\title{
MEMANTINE PREVENTS CARDIOMYOCYTES NUCLEAR SIZE REDUCTION IN THE LEFT VENTRICLE OF RATS EXPOSED TO COLD STRESS
}

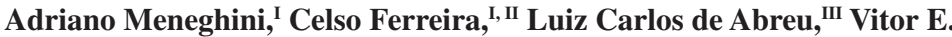

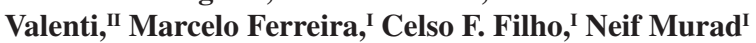

doi: $10.1590 / \mathbf{S 1 8 0 7 - 5 9 3 2 2 0 0 9 0 0 0 9 0 0 0 1 4}$

Meneghini A, Celso Ferreira, Luiz Carlos de Abreu, Vitor E. Valenti, Marcelo Ferreira, Celso F. Filho, Neif Murad. Memantine prevents nuclear size reduction in cardiomyocytes in the left ventricle of rats exposed to cold stress. Clinics. 2009;64(9):921-6.

OBJECTIVES: Memantine is an N-methyl-d-aspartate (NMDA) glutamate receptor antagonist used to treat Alzheimer's disease. Previous studies have suggested that receptor blockers act as neuroprotective agents; however, no study has specifically investigated the impact that these drugs have on the heart. We sought to evaluate the effects of memantine on nuclear size reduction in cardiac cells exposed to cold stress.

METHOD: We used male EPM-Wistar rats ( $n=40)$ divided into 4 groups: 1) Matched control (CON); 2) Memantine-treated rats (MEM); 3) Rats undergoing induced hypothermia (IH) and 4) Rats undergoing induced hypothermia that were also treated with memantine (IHM). Animals in the MEM and IHM groups were treated by oral gavage administration of $20 \mathrm{mg} / \mathrm{kg} / \mathrm{day} \mathrm{memantine}$ over an eight-day period. Animals in the IH and IHM groups were submitted to 4 hours of hypothermia in a controlled environment with a temperature of $-8^{\circ} \mathrm{C}$ on the last day of the study.

RESULTS: The MEM group had the largest cardiomyocyte nuclear size $\left(151 \pm 3.5 \mu \mathrm{m}^{3}\right.$ vs. CON: $142 \pm 2.3 \mu \mathrm{m}^{3}$; $\left.\mathrm{p}<0.05\right)$, while the IH group had the smallest mean value of nuclear size. The nuclear size of the IHM group was preserved $\left(125 \pm 2.9 \mu \mathrm{m}^{3}\right) \mathrm{compared}$ to the IH group $\left(108 \pm 1.7 \mu \mathrm{m}^{3} ; \mathrm{p}<0.05\right)$.

CONCLUSION: Memantine prevented the nuclear size reduction of cardiomyocytes in rats exposed to cold stress.

KEYWORDS: Memantine; Cardiac myocytes; Cardiotonic agents; Cell nucleus structures; Heart.

\section{INTRODUCTION}

Reduced body temperature induced by exposure to a cold surrounding environment is considered a physiological stressor. ${ }^{1-3}$ Neurogenic lesions related to myocardial hypertrophy and changes in myocardial tissue metabolism ${ }^{4}$ can be also caused by the reaction of cardiovascular tissue to cold stress, and these lesions are hypothesized

I Departamento de Clínica Médica, Disciplina de Cardiologia, Faculdade de Medicina do ABC - Santo André/SP, Brasil.

II Departamento de Medicina, Disciplina de Cardiologia, Universidade Federal de São Paulo (UNIFESP) - São Paulo/SP, Brazil.

III Departamento de Saúde Pública, Universidade de São Paulo - São Paulo/ SP, Brasil.

Email: celsoferreira.dmed@epm.br

Tel: 55114993.5403

Received for publication on May 18, 2009

Accepted for publication on June 24, 2009 to be a marker for subsequent cardiovascular disease as well as a predictor of hypertension. ${ }^{5}$ Recently, this model was used to investigate several aspects of cardiac injury. ${ }^{6}$ Interestingly, a previous study indicated that cold stress acutely induces a reduction in the nuclear size (hence atrophy) of cardiomyocytes in rats, ${ }^{7}$ which is in contrast to the finding that is observed in myocardial hypertrophy. This reduction in nuclear size has been hypothesized to be a marker of ischemia during periods of physiological stress. ${ }^{7}$

It has been well established that glutamate excitotoxicity triggers neurodegeneration in patients with medical conditions that can lead to acute brain injuries such as stroke, status epilepticus or head trauma. Drugs that block N-methyl-daspartate (NMDA) glutamate receptors have been shown to be neuroprotective in animal models of these medical conditions. ${ }^{8,9}$ Memantine is a non-competitive antagonist of NMDA receptors and is currently used to treat patients 
with moderate to severe Alzheimer's disease to improve cognition. ${ }^{10,12}$ Its mechanism of action is thought to be related to its effects on $\mathrm{Ca}^{2+}$ homeostasis. An increase in cytosolic Calcium $\left(\mathrm{Ca}^{2+}\right)$ is associated with functional impairment of many organelle and is also strongly associated with apoptosis. ${ }^{13}$ Previous studies have provided evidence that memantine is able to prevent ischemic injuries in the retina and in neurons exposed to different aggressive agents (that lead to increases in cytosolic $\mathrm{Ca}^{2+}$ ) due its effects on $\mathrm{Ca}^{2+} \cdot{ }^{14,15}$

Although various studies have suggested that NMDA receptor antagonists have neuroprotective properties, no previous study has specifically investigated the influence this class of drugs may have on the heart. Previous studies, however, have reported an association between memantine and the prevention of ischemia under conditions of cellular stress. ${ }^{14,16}$ Therefore, we sought to evaluate the effects of memantine on the nuclear size of cardiomyocytes in the left ventricle of rats exposed to cold stress.

\section{METHODS}

\section{Animals}

Experiments were conducted on forty adult male Wistar rats that weighed $200-250 \mathrm{~g}$. The ambient temperature of the environment in which the rats were housed was $22^{\circ} \mathrm{C}$, with the humidity at $\sim 60 \%$. The rats were kept on a 12 -hour light/12-hour dark cycle. Animals had free access to food and water. Animals were randomized into four groups: 1) the control group (CON, $n=10)$, in which rats were treated with gavage administration of $1 \mathrm{~mL}$ of water for eight consecutive days; 2) the memantine group (MEM, $n=10)$, in which rats were treated with gavage administration of water $(1 \mathrm{~mL})$ containing $20 \mathrm{mg} / \mathrm{kg}$ of memantine for eight consecutive days; 3 ) the induced hypothermia group $(\mathrm{IH}, \mathrm{n}=10)$, in which rats were treated with gavage administration of $1 \mathrm{~mL}$ of water for eight consecutive days and exposed to a $-8^{\circ} \mathrm{C}$ environment for four hours on the last $\left(8^{\text {th }}\right)$ day of the study and; 4) the memantine plus induced hypothermia group (IHM, $n=10$ ), in which rats were treated with gavage administration of water (1 mL) containing $20 \mathrm{mg} / \mathrm{kg}$ memantine for eight consecutive days and exposed to $\mathrm{a}-8^{\circ} \mathrm{C}$ environment for four hours on the last $\left(8^{\text {th }}\right)$ day of the study. All experiments were performed in accordance with the ethical guidelines of the National Institutes of Health Guide for the Care and Use of Laboratory Animals and were approved by the Ethical Committee for research at our University (number 003/08).

\section{Induced Hypothermia Procedure}

Rats were exposed to cold stress, which was induced by placing them in wire mesh cages in an open refrigerated compartment at $-8^{\circ} \mathrm{C}$ for 4 hours. Rats were exposed to this environment only once and their behavior was observed throughout the stress experiment ${ }^{7}$. Rats' body temperatures were monitored and maintained near $37^{\circ} \mathrm{C}$. No rats died during the induced hypothermia procedure.

\section{Histological Examination}

To verify if the exposure to cold stress was sufficient to cause stress responses characterized by lipid and glycogen depletion in adrenal gland and liver, we evaluated lipid depletion in adrenal gland cortical cells and glycogen depletion in hepatocytes. After administration of an adequate level of ether anesthesia, we examined the rats' tail tone and response to external stimuli before and during surgical procedure through the evaluation of vibrissa movements. All an

imals were then submitted to laparotomy. Two pieces of the left lobe of the liver and right adrenal gland were removed for microscopy investigation. These specimens were cut into small pieces $\left(1 \mathrm{~mm}^{3}\right)$, post-fixed in $1 \% \mathrm{OsO}_{4}$ solution for 2 hours, dehydrated and embedded in araldite. Silver or gray thin sections $(60-90 \mathrm{~nm})$ were produced using a Porter-Blum MT-B ultramicrotome. The sections were then mounted on copper silver grids with 200 patches and stained with uranyl acetate and lead citrate. We presented data regarding lipid depletion in adrenal gland cortical cells and glycogen depletion in hepatocytes, respectively, as staining intensity levels (+=small intensity; $++++=$ high intensity). ${ }^{6}$

\section{Nuclear Volume Measurement}

All animals were also submitted to thoracotomy. The thorax of each rat was opened and the left ventricle was exposed and removed. Fragments of heart material were fixed in Bouin's solution, mounted and paraffinated. Sections measuring $10 \mu \mathrm{m}$ were stained with hematoxylineosin. In order to estimate the nuclear size of each cell, karyometry was used according to the same principles described in a previous publication. ${ }^{17}$ Morphometric evaluation was performed using the Quantimet Color Option (Leica, Cambridge) image analysis system. Measurements of cardiomyocyte nuclear parameters were performed exclusively on clearly visible longitudinal sections of muscle fibers in which cardiomyocyte nuclei had a clear outline. To calculate nuclear volume, we used the following equation proposed by Salvatore: ${ }^{17}$

$\mathrm{V}=\left(\mathrm{A}^{2} \times \mathrm{B}\right) / \mathbf{1 . 9 1}$

$\mathrm{V}=$ volume; $\mathrm{A}=$ smaller axial measure; $\mathrm{B}=$ bigger axial measure; $1.91=$ constant. 
Using a low power field diameter of $1800 \mu \mathrm{m}$, outlines of 150 nuclei were drawn for each sample by camera lucida. Arithmetical means of the diameters of nuclei were grouped into frequency classes. The samples were examined by three independent investigators using standardized criteria. ${ }^{7}$

\section{Statistical Analysis}

The results are reported as means \pm standard deviations. In order to examine cell volume data, the KruskalWallis and Tukey post-hoc tests were applied to allow comparisons between independent groups. The concordance of the measurements performed by the three individual investigators was evaluated and analyzed using Bartko's intra-class correlation coefficient according to the Fleiss guidelines. ${ }^{17}$ The significance level was set at $\mathrm{R}>0.75$ or $\mathrm{p}<0.05$.

\section{Bartko's test formula}

$R=\frac{N(P M S-E M S)}{N(P M S)+(K-1)(R M S)+(N-1)(K-1) E M S}$

R- Bartko's correlation index

PMS- Patients Mean Square

RMS- Researcher Mean Square

EMS- Error Mean Square

N- Number of events

K- Number of investigators

\section{RESULTS}

We noted the following reponses in rats after cold stress exposure: hair bristling, paw edema, tremor, tail stretching and tachypnea. There were no response differences noted between the memantine-treated group and the untreated group. Body weights were not statistically different among the groups (IH group: $306.2 \pm 20.3$ grams; CON group: $308.6 \pm 29.7$ grams; MEM group: $265.8 \pm 29.4$ grams; IHM group: 254.6 \pm 21.9 grams).

To validate whether or not exposure to cold stress was sufficient to cause stress response, we examined lipid depletion in adrenal gland cells (Table 1) and glycogen depletion in hepatocytes (Table 2) of rats from each group.

The IH group had highest rate of lipid depletion in adrenal gland cells $(\mathrm{p}<0.05)$ and the highest rate of glycogen depletion in hepatocytes $(\mathrm{p}<0.05)$, which supports our hypothesis that exposure to $-8^{\circ} \mathrm{C}$ for four hours was effective at inducing physiological stress.

The rats in the IH group had a smaller cardiomyocyte nuclear size (Figure 1) compared to the other three groups $(\mathrm{p}<0.05)$. Our analysis revealed that the rats in the IHM
Table 1 - Lipid depletion intensity in adrenal gland cortical cells stained with Sudan IV* (+=small intensity; ++++=high intensity)

\begin{tabular}{lcccc}
\hline Animal & Cold Stress & Control & Memantine & $\begin{array}{c}\text { Memantine }+ \\
\text { Cold Stress }\end{array}$ \\
\hline 1 & ++ & ++++ & ++++ & ++ \\
2 & ++ & +++ & +++ & +++ \\
3 & + & ++++ & +++ & ++ \\
4 & ++ & ++++ & +++ & + \\
5 & +++ & ++++ & ++++ & +++ \\
6 & + & ++++ & +++ & + \\
7 & + & ++ & +++ & ++ \\
8 & + & +++ & ++ & +++ \\
9 & + & ++++ & ++++ & ++ \\
10 & + & +++ & +++ & ++ \\
$*$ & \\
lipid depletion) & & &
\end{tabular}

Table 2 - Glycogen depletion intensity in hepatocytes blushed with PAS method* (+=small intensity; ++++=high intensity)

\begin{tabular}{lcccc}
\hline Animal & Cold Stress & Control & Memantine & $\begin{array}{c}\text { Memantine }+ \\
\text { Cold Stress }\end{array}$ \\
\hline $\boldsymbol{1}$ & ++ & ++++ & ++++ & ++ \\
2 & ++ & ++++ & ++++ & ++ \\
3 & +++ & ++++ & +++ & ++ \\
4 & ++ & +++ & +++ & +++ \\
5 & ++ & ++++ & +++ & ++ \\
6 & + & ++++ & ++++ & ++ \\
7 & + & ++ & ++++ & ++ \\
8 & + & ++ & +++ & +++ \\
9 & + & +++ & ++++ & ++ \\
10 & ++ & ++ & ++++ & ++ \\
\hline
\end{tabular}

$*_{+}=$Low intensity (more glycogen depletion); ++++ = High intensity (less glycogen depletion)

group had a larger nuclear size than rats in the IH group, likely because the memantine treatment provided protection on the ultrastructural level $(\mathrm{p}<0.05)$.

We compared teh groups on the basis of nuclear size (Figure 1). The nuclear size of the IH group (Figure 2A) was significantly decreased as compared to the control group (Figure 2B) $(\mathrm{p}<0.05)$. Furthermore, rats in the IHM group (Figure 2C) had a $76 \%$ reduction in their cardiomyocyte nuclear size as compared to rats stressing the MEM group (Figure 2D) $(\mathrm{p}<0.05)$. Data variance analysis using Bartko's correlation index yielded results ranging from $0.44-0.96$ in all experimental groups, thus validating our methodology. Furthermore, when we performed variation analysis 


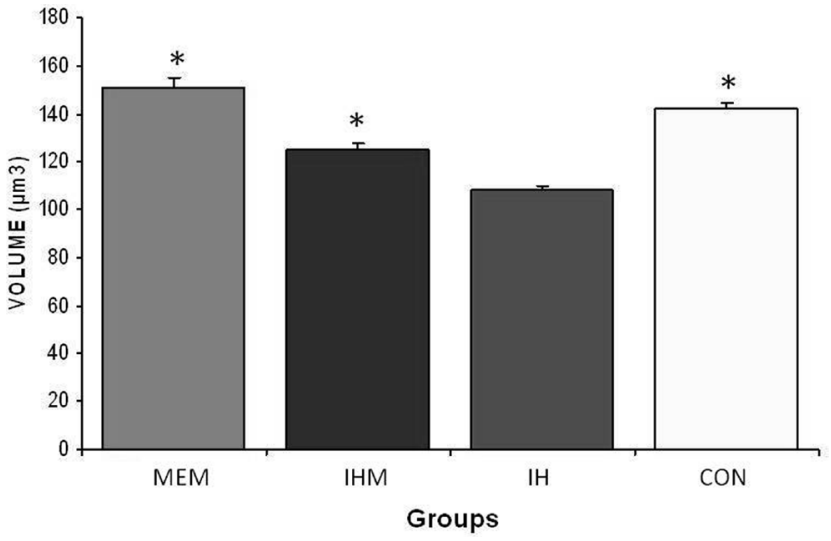

Figure 1 - The nuclear size of cardiomyocytes in the control (CON), memantine-treated (MEM), induced hypothermic/memantine-treated (IHM) and induced hypothermic (IH) groups. $* \mathrm{p}<0.05$ for $\mathrm{IH}$ as compared to the other groups

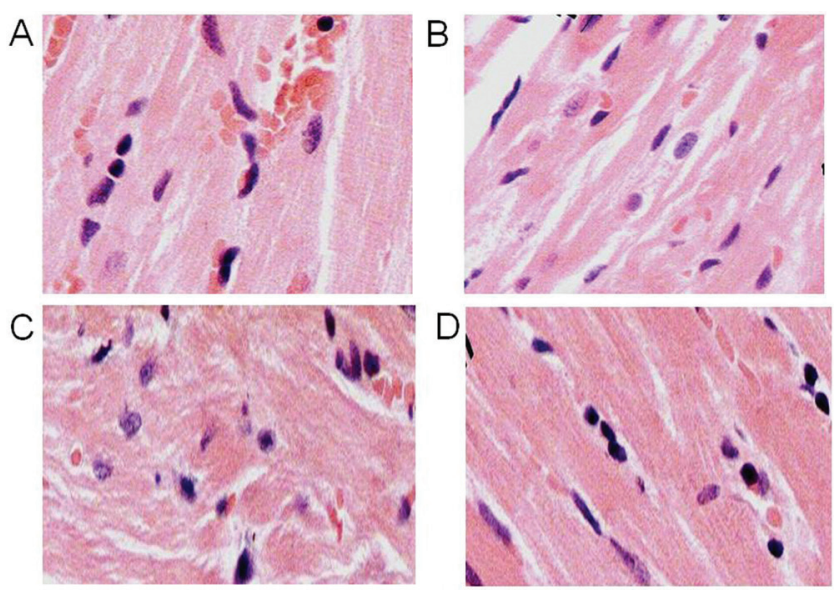

Figure 2 - In the IH group (A), cardiomyocyte nuclei were found to be smaller and have a lower volume than the nuclei in other groups. Cardiomyocytes in the $\mathrm{CON}$ group (B) had normal cardiomyocyte nuclear size without evidence of any type of cellular injury. In the IHM group (C) decreased cardiomyocyte nuclear volume was observed, but the nuclei were larger than those of cardiomyocytes in the IH group. In the MEM group, (D) we observed increased cardiomyocyte nuclear size. The nuclei were normal in appearance without evidence any type of cellular injury (HE - Amplification $400 \mathrm{x}$ )

examining group mean differences, we found a statistically significant difference between groups $(\mathrm{p}<0.05)$.

\section{DISCUSSION}

We sought to evaluate the effects of memantine administration on the heart tissue of rats exposed to cold stress. As demonstrated in a previous study, ${ }^{7}$ we found that exposure to cold stress for four hours at $-8^{\circ} \mathrm{C}$ decreased cardiomyocyte nuclear size in rats. We also verified that rats in the group treated with memantine had the largest mean cardiomyocyte nuclear size of all groups in this study. Moreover, rats treated with memantine and exposed to cold stress were found to have a larger cardiomyocyte nuclear size than rats that were exposed to cold stress but not treated with memantine.

Our investigation demonstrated that induced hypothermia causes cardiomyocyte nuclear volume reduction. According to prior research, physiological stressors such as exercise, fasting and cold are known triggers of different physiological pathways through mechanisms that are not yet completely understood. ${ }^{19}$ In a study performed by Matsuoka et al. ${ }^{20}$, it was observed that the cellular stress caused by hypothermic exposure exerted a lethal effect on hamsters with cardiomyopathy and therefore suggested that cold stress leads to, or increases cardiovascular dysfunction in animals. Previous studies have shown that cold stress triggers activation of a mitochondria-dependent signaling pathway in cardiomyocytes, which is an important finding, given that mitochondrial stress either leads to cellular adaptation and survival or induces apoptotic signaling in various types of cells. ${ }^{6,21,22}$ Thus, the increase in metabolic load and reduction in ATP production caused by stress cause a cytosolic $\mathrm{Ca}^{2+}$ imbalance that leads to hypoxia and irreversible cellular injury. ${ }^{23}$ Our histological analysis, which showed an increased rate of lipid depletion in the adrenal gland and a higher rate of glycogen depletion in liver cells, supports these recent findings.

We observed that rats in the IHM group had a larger nuclear size than rats in the IH group. However, we cannot confirm the effects that memantine administration has on cardiovascular parameters identified in prior studies because we did not examine hemodynamic determinants. Several prior studies have described the association between memantine and the prevention of ischemia. ${ }^{13,15}$ Osborne et al. ${ }^{14}$ examined whether or not memantine could slow down the changes observed in rat retina following ischemia and reperfusion. When memantine $(5 \mathrm{mg} / \mathrm{kg})$ was given at the onset of ischemia, it prevented ischemia/reperfusion injuries to varying degrees. However, when memantine was given at the onset of the reperfusion, this was not observed. The authors therefore suggested that a single injection of memantine given intraperitoneally or intravitreally protects the retina from a subsequent ischemic insult. D'Amico et al. ${ }^{16}$ observed that the incidence of ventricular tachycardia, ventricular fibrillation and mortality induced by reperfusion was greatly reduced in animals treated with intravenous memantine $(1.5 \mathrm{mg} /$ $\mathrm{kg}$ ) injected five minutes prior to coronary artery occlusion $(\mathrm{p}<0.01)$. They also indicated that reperfusion-induced arrhythmias, but not ischemia-induced arrhythmias, were preventable by administration of both NMDA and non-NMDA ionotropic excitatory amino acid receptor antagonists.

In our study, Bartko's intra-class correlation coefficient revealed that there was significant concordance among the investigators. The mean indexes of intra-class correlation 
(R) ranged between 0.44 and 0.96 for each animal studied. Statistically significant differences were demonstrated between all groups, and the results were complemented by a group mean difference analysis. Therefore, based on our statistical analyses, we were able to validate the reliability of this method for assessing differences among groups.

The effect that memantine was found to have on the cardiac cells in our study may lead to new perspectives regarding its role in the cardiovascular system. Recent studies have demonstrated the association between memantine and various cardiovascular parameters. Lapchak et al. ${ }^{24}$ examined whether or not the manipulation of oxidative stress and components of the blood coagulation cascade might lead to an improved prognosis in patients that have had spontaneous hemorrhages. The authors suggested that promising drugs that may be considered for use in combination therapy include non-competitive NMDA receptor antagonists, such as memantine, which have been shown to reduce hemorrhage and behavioral deficits in animal models. The mechanism for this activity is not well understood. Collins et al. ${ }^{25}$ revealed that left ventricular peak and end-diastolic pressures following cocaine use were greater in individuals treated with memantine, although these elevations were not clinically significant. In another study examining memantine's effect on cardiovascular parameters,
Herrero et al. ${ }^{26}$ found that memantine did not significantly affect blood pressure.

To our knowledge, there has been no prior study that has examined the association between memantine administration and alterations in cardiomyocyte nuclear size. We found that memantine was an effective $\mathrm{Ca}^{2+}$ channel blocker in cardiac myocytes, which is evidenced by the preservation of nuclear size that occurred in this rat model of cardiac injury. In addition, an unexpected observation made in this study was that the group treated with memantine had the largest nuclear size, which supports the idea that memantine may also act as a cardioprotective drug. This is the first study to demonstrate that this drug preserves the nuclear size of cardiomyocytes in animals that have been exposed to cold stress. We believe that these data are important in developing future therapies to preserve cardiomyocyte structure under conditions of cold stress.

In summary, our results indicate that memantine prevents cardiomyocyte nuclear size reduction in the left ventricles of rats exposed to cold stress.

\section{ACKNOWLEDGMENT}

We thank Mr. Brandon Saltzgiver for his critical evaluation of English Grammar.

\section{REFERENCES}

1. Rintamaki H, Rissanen S. Heat strain in cold. Ind Health. 2006;44:42732.

2. Andraus W, Jukemura J, Dutra F, Bechara E, Cunha JE, Leite KR, et al. Oxidative stress is enhanced by hypothermia imposed on ceruleininduced pancreatitis in rats. Clinics. 2007;62:483-90.

3. de Assis MA, de Mello MF, Scorza FA, Cadrobbi MP, Schooedl AF, da Silva SG, et al. Evaluation of physical activity habits in patients with posttraumatic stress disorder. Clinics. 2008;63:473-8.

4. Cox BF, Bishop VS. Neural and humoral mechanisms of angiotensindependent hypertension. Am J Physiol. 1991;261:H1284-H1291.

5. Machado BH, Brody MJ. Effect of nucleus ambiguus lesion on the development of neurogenic lesion. Hypertension. 1998;11:I135-I38.

6. Bombig MT, Ferreira C, Mora O, Soares JD, Póvoa R, Luna Filho B, et al. Pravastatin protection from cold stress in myocardium of rats. Jpn Heart J. 2003;44:243-55.

7. Meneghini A, Ferreira C, Abreu LC, Ferreira M, Ferreira Filho C, Valenti VE, et al. Cold stress effects on cardiomyocytes nuclear size in rats: light microscopic evaluation. Rev Bras Cir Cardiovasc. 2008;23:530-3.

8. Olney JW, Labruyere J, Wang G, Wozniak DF, Price MT, Sesma MA. NMDA antagonist neurotoxicity: mechanism and prevention. Science. 1991;254:1515-8.
9. Choi DW. Excitotoxic cell death. J Neurobiol. 1992;23:1261-76.

10. Lipton SA. Failures and successes of NMDA antagonists: molecular basis for the use of open-channel blockers like memantine in treatment of acute and chonic neurological insults. NeuroRx. 2004;1:101-10.

11. Areosa SA, Sherriff F. Memantine for dementia. Cochrane Database Syst Rev. 2003;3: CD003154.

12. Reisberg B, Doody R, Stoffler A. Memantine in moderate-to-severe Alzheimer's disease. N Engl J Med. 2003;348:1333-41.

13. Bojarski L, Herms J, Kuznicki J. Calcium dysregulation in Alzheimer's disease. Neurochem Int. Neurochem Int. 2008;52:621-33.

14. Osborne NN. Memantine reduces alterations to the mammalian retina, in situ, induced by ischemia. Vis Neurosci. 1999;16:45-52.

15. Tozzi A, Costa C, Di Filippo M, Tantucci M, Siliquini S, Belcastro V et al. Memantine reduces neuronal dysfunctions triggered by in vitro ischemia and 3-nitropropionic acid. Exp Neurol. 2007;207:218-26.

16. D’Amico M, Di Filippo C, Rossi F, Rossi F. Arrhythmias induced by myocardial ischaemia-reperfusion are sensitive to ionotropic excitatory amino acid receptor antagonists. Eur J Pharmacol. 1999;366:16774.17. Salvatore CA. A cytological examination of uterine growth during the estrous cycle and artifically induced estrus. Rev Bras Biol. 1948;8:505-23. 
18. Fleiss JL, Spitzer RL, Cohen J, Endicott J. Three computer diagnosis methods compared. Arch Gen Psychiatry. 1972;27:643-9.

19. Eliot RS. Stress and cardiovascular disease: mechanisms and measurement. Ann Clin Res. 1987;19:88-95.

20. Matsuoka N, Arakawa H, Kodama H, Yamaguchi I. Characterization of stress-induced sudden death in cardiomyopathic hamsters. J Pharmacol Exp Ther. 1998;284:125-35.

21. Siu PM, Pistilli EE, Ryan MJ, Alway SE. Aging sustains the hypertrophyassociated elevation of apoptotic suppressor X-linked inhibitor of apoptosis protein (XIAP) in skeletal muscle during unloading. J Gerontol A Biol Sci Med Sci. 2005;60:976-83.

22. Ferri KF, Jacotot E, Leduc P, Geuskens M, Ingber DE, Kroemer G. Apoptosis and karyogamy in syncytia induced by the HIV-1-envelope glycoprotein complex. Cell Death Differ. 2000;7:1137-9.
23. Kutala VK, Khan M, Angelos MG, Kuppusamy P. Role of oxygen in postischemic myocardial injury. Antioxid Redox Signal. 2007;9:1193206.

24. Lapchak PA, Araujo DM. Advances in hemorrhagic stroke therapy: conventional and novel approaches. Expert Opin Emerg Drugs. 2007;12:389-406.

25. Collins ED, Vosburg SK, Ward AS, Haney M, Foltin RW. Memantine increases cardiovascular but not behavioral effects of cocaine in methadone-maintained humans. Pharmacol Biochem Behav. 2006;83:47-55.

26. Herrero JF, Headley PM, Parsons CG. Memantine selectively depresses NMDA receptor-mediated responses of ratspinal neurones in vivo. Neurosci Lett. 1994;165:37-40. 